\title{
New Algorithm for Minimax Design of Sparse IIR Filters
}

\author{
Wu-Sheng $\mathrm{Lu}$ \\ Dept. of Electrical and Computer Engineering \\ University of Victoria \\ Victoria, BC, Canada V8W 3P6 \\ Email: wslu@ece.uvic.ca
}

\author{
Takao Hinamoto \\ Graduate School of Engineering \\ Hiroshima University \\ Higashi-Hiroshima, 739-8527, Japan \\ Email: hina@crest.ocn.ne.jp
}

\begin{abstract}
Sparse digital filters are of importance as they offer improved implementation efficiency relative to their nonsparse counterparts. This paper examines the design of minimax sparse IIR filters from a sparse representation point of view. The result is a new algorithm that accomplishes a design with three phasesidentification of the whereabouts of zero coefficients; optimal design subject to the sparsity constraint; and performance enhancement by further dimension reduction of the working subspace. A design example is presented for illustrating the new algorithm.
\end{abstract}

\section{INTRODUCTION}

Digital filters with sparse coefficients are of practical importance as they offer improved implementation efficiency relative to their nonsparse counterparts. Research in this area has been active since 1990's [1]-[4]. The surge of research in compressive sensing (CS) over the past several years is largely responsible for the recent renewed activities in sparse filters [5]-[13] due primarily to the fact that sparsity plays a key role in the CS theory [14] and methods developed in CS based on $l_{1}$-minimization and matching pursuit [15] for the recovery of sparse signals are applicable to sparse filter designs.

To date, most of the work in this area has been focused on sparse FIR filters, except that of [9]. This paper presents a new algorithm for minimax design of stable sparse IIR filters. The method is different from that of [9] in several ways. First, the new algorithm is built on a linear sparse representation framework. This linear representation is made possible due to the fact that the poles of a sparse IIR filter are very close to those of a nonsparse IIR counterpart as long as both filters approximate the same frequency response, which is in a certain sense similar to an observation made in [16]. Second, here we adopt a constrained formulation to avoid the use of a regularization parameter as in [9]. In this way, the filter's sparsity is explicitly related to the minimax error of the filter, which in turn makes sparsity tuning easier and more intuitive. Third, the new algorithm includes an additional phase to enhance performance by further reduction of the working subspace's dimension and re-optimization. A design example is presented to illustrate the new algorithm.

\section{DESIGN FROM A SPARSE REPRESENTATION PERSPECTIVE}

Consider an IIR transfer function of order $(n, r)$, which assumes the form

$$
H(z)=\frac{a(z)}{b(z)}=\frac{\sum_{k=0}^{n} a_{k} z^{-k}}{\sum_{i=0}^{r} b_{i} z^{-i}} \quad \text { with } b_{0}=1
$$

If we temporarily fix $b(z)$, we can write the filter's frequency response as

$$
H\left(e^{j \omega}\right)=\sum_{k=0}^{n} a_{k} w_{k}(\omega)
$$

where $w_{k}(\omega)$ are known functions and given by

$$
\begin{aligned}
w_{k}(\omega) & =u_{k}(\omega)-j v_{k}(\omega) \\
u_{k}(\omega) & =\frac{b_{r}(\omega) \cos k \omega+b_{i}(\omega) \sin k \omega}{p(\omega)} \\
v_{k}(\omega) & =\frac{b_{r}(\omega) \sin k \omega-b_{i}(\omega) \cos k \omega}{p(\omega)} \\
p(\omega) & =b_{r}^{2}(\omega)+b_{i}^{2}(\omega) \\
b_{r}(\omega) & =\sum_{i=0}^{r} b_{i} \cos i \omega, b_{i}(\omega)=\sum_{i=0}^{r} b_{i} \sin i \omega
\end{aligned}
$$

When it comes to sparse IIR filters, the designer is primarily interested in having a sparse set of coefficients $\left\{a_{k}\right\}$ while $\left\{b_{k}\right\}$ remains nonsparse because the denominator $b(z)$ is often of relatively low order and is too critical to impose additional constraints such as sparsity in addition to stability. In the light of (2), therefore, designing a sparse IIR filter amounts to finding a sparse representation of frequency response $H\left(e^{j \omega}\right)$ in the space spanned by "basis" functions $\left\{w_{k}(\omega), k=0,1, \ldots, n\right\}$ so as to best approximate a desired frequency response $H_{d}(\omega)$ over a frequency region of interest $\Omega \subset[0, \pi]$.

For a minimax design of order $(n, r)$ and a lower bound of sparsity $K$, one seeks to find an $H(z)$ in (1) that solves the problem

$$
\begin{array}{lll}
\underset{\boldsymbol{a}, \boldsymbol{b}}{\operatorname{minimize}} & \underset{\omega \in \Omega}{\operatorname{maximize}} & \left|H\left(e^{j \omega}\right)-H_{d}(\omega)\right| \\
\text { subject to: } & \text { number of zero } a_{k}^{\prime} s \geq K \\
& H(z) \text { is stable }
\end{array}
$$

where $\boldsymbol{a}=\left[\begin{array}{llll}a_{0} & a_{1} & \ldots & a_{n}\end{array}\right]^{T}, \boldsymbol{b}=\left[\begin{array}{llll}b_{1} & b_{2} & \ldots & b_{r}\end{array}\right]^{T}$, and $H\left(e^{j \omega}\right)$ in (4a) assumes the form of (2).

\section{THE DESIGN METHOD}

\section{A. Linear representation of $H\left(e^{j \omega}\right)$}

Given a desired frequency response $H_{d}(\omega)$ and order $(n, r)$, minimax design of a conventional (i.e. nonsparse) stable IIR filter can readily be carried out [15, Ch. 16]. In our design, the denominator obtained, denoted by $b(z)$, is utilized in (3) 
to generate the basis functions $\left\{w_{k}(\omega), k=0,1, \ldots, n\right\}$ in (2). There are two reasons for using a fixed denominator in our design: First, from (3a)-(3e) it follows that a fixed $b(z)$ leads to a fixed set of basis functions $\left\{w_{k}(\omega)\right\}$, hence a linear representation of frequency response $H\left(e^{j \omega}\right)$ in terms of $\left\{w_{k}(\omega)\right\}$ as seen in (2). This linear representation is the foundation on which the new design method is developed, see the rest of the section for details. Second, our design practice based on the new algorithm has indicated that the poles of the sparse filter designed (with both the coefficients of $a(z)$ and $b(z)$ being treated as design variables) practically do not move when compared with the poles of $b(z)$ obtained from a nonsparse design, especially when the order of $b(z)$ is not high. We remark that this phenomenon coincides with an observation made in [16], although [16] does not deal with sparse filters.

\section{B. Phase 1-Identifying indices of zero coefficients}

With a linear representation of $H\left(e^{j \omega}\right)$ in the space spanned by $\left\{w_{k}(\omega)\right\}$ in place, phase 1 of the design identifies an index set $\mathcal{I}^{*}$ with length $\left|\mathcal{I}^{*}\right| \geq K$ for coefficients $\left\{a_{k}\right\}$ that are most appropriate to be set to zero. Here the term "most appropriate" is understood in a design context, thus it refers to those coefficients whose nullification shall lead to least degradation in the filter's performance. To this end, we seek a sparse $\left\{a_{k}\right\}$ subject to sufficient closeness between $H\left(e^{j \omega}\right)$ and $H_{d}(\omega)$ across the entire region $\Omega$. Note that stability is not an issue here because a stable and fixed $b(z)$ has been used in constructing the basis functions $\left\{w_{k}(\omega)\right\}$ (see Sec. 3.A). The index identification problem at hand is formulated as

$$
\begin{array}{cl}
\text { minimize } & \|\boldsymbol{a}\|_{1} \\
\text { Subjiect to: } & \left|\sum_{k=0}^{n} a_{k} w_{k}(\omega)-H_{d}(\omega)\right| \leq \delta \text { for } \omega \in \Omega
\end{array}
$$

where $\delta>0$ is a small constant to be specified later and $\|\boldsymbol{a}\|_{1}=\sum_{i=0}^{n}\left|a_{i}\right|$ is the $l_{1}$-norm of $\boldsymbol{a}$ whose minimization promotes a sparse coefficient vector $\boldsymbol{a}$ subject to constraint (5b) [14].

Since $\|\boldsymbol{a}\|_{1}$ is a convex function of $\boldsymbol{a}$ and the constraints in (5b) are linear, (5) is a convex problem which can be solved as a second-order cone programming (SOCP) problem as argured below.

Let $H_{d}(\omega)=H_{d r}(\omega)-j H_{d i}(\omega), \quad \boldsymbol{h}_{d}(\omega)=$ $\left[\begin{array}{lll}H_{d r}(\omega) & H_{d i}(\omega)\end{array}\right]^{T}, \boldsymbol{u}(\omega)=\left[\begin{array}{llll}u_{0}(\omega) & u_{1}(\omega) & \ldots & u_{n}(\omega)\end{array}\right]^{T}$, $\boldsymbol{v}(\omega)=\left[\begin{array}{llll}v_{0}(\omega) & v_{1}(\omega) & \ldots & v_{n}(\omega)\end{array}\right]^{T}$. From (3) it follows that

$$
\begin{aligned}
\left|\sum_{i=0}^{n} a_{k} w_{k}(\omega)-H_{d}(\omega)\right| & =\left\|\left[\begin{array}{l}
\boldsymbol{u}^{T}(\omega) \\
\boldsymbol{v}^{T}(\omega)
\end{array}\right] \boldsymbol{a}-\boldsymbol{h}_{d}(\omega)\right\|_{2} \\
& =\left\|\boldsymbol{S}(\omega) \boldsymbol{a}-\boldsymbol{h}_{d}(\omega)\right\|_{2}
\end{aligned}
$$

where $\boldsymbol{S}(\omega)=\left[\begin{array}{l}\boldsymbol{u}^{T}(\omega) \\ \boldsymbol{v}^{T}(\omega)\end{array}\right]$. Let $\left\{\omega_{1}, \omega_{2}, \ldots, \omega_{L}\right\}$ be a dense and evenly distributed frequency grids over $\Omega$, it follows from (6) that constraint (5b) may be realistically replaced by

$$
\left\|\boldsymbol{S}\left(\omega_{l}\right) \boldsymbol{a}-\boldsymbol{h}_{d}\left(\omega_{l}\right)\right\|_{2} \leq \delta \quad l=1,2, \ldots, L
$$

The objective function $\|\boldsymbol{a}\|_{1}$ is convex but non-differentiable. To deal with this nonsmoothness, an upper bound $\sigma_{i}$ for the magnitude of each component of $\boldsymbol{a}$ is introduced as a new design variable. In this way, problem (5) can be re-formulated as

$$
\begin{array}{cl}
\underset{a, \sigma}{\operatorname{minimize}} & \sum_{i=0}^{n} \sigma_{i} \\
\text { subject to } & \left|a_{i}\right| \leq \sigma_{i} \quad i=0,1, \ldots, n \\
& \left\|\boldsymbol{S}\left(\omega_{l}\right) \boldsymbol{a}-\boldsymbol{h}_{d}\left(\omega_{l}\right)\right\|_{2} \leq \delta \\
& \text { for } l=1,2, \ldots, L
\end{array}
$$

which is a standard SOCP problem [17] that can be solved by using an efficient convex programming solver such as SeDuMi.

The solution of problem (8) is sparse in general, as long as the feasible region defined by (8b) and (8c) is nonempty. The question is whether or not the sparsity constraint (4b) is met by the solution of (8). It is in this regard the error bound $\delta$ in (8c) plays a role: a greater $\delta$ defines a larger feasible region which includes increasing number of sparser solutions. Therefore, the designer may use $\delta$ as a means to control the degree of sparsity in coefficients $\left\{a_{k}\right\}$ so as to satisfy (4b).

We remark that, in addition to using $\delta$ to control solution's sparsity, hard-thresholding with an appropriate threshold value may be applied to the solution of (8) as a final step for fine tuning the exact number of zero coefficients in $\left\{a_{k}\right\}$. In summary, the result of design phase 1 is an index set $\mathcal{I}^{*} \subset\{0,1, \ldots, n\}$ with $\left|\mathcal{I}^{*}\right| \geq K$ for nullifying coefficients $\left\{a_{k}, k \in \mathcal{I}^{*}\right\}$.

\section{Phase 2-Optimum design of sparse IIR filters}

With index $\mathcal{I}^{*}$ identified, we now revisit problem (4) which can now be expressed more specifically as

$$
\begin{aligned}
\underset{\boldsymbol{a}, \boldsymbol{b}}{\operatorname{minimize}} \underset{\omega \in \Omega}{\operatorname{maximize}} & \left|H\left(e^{j \omega}\right)-H_{d}(\omega)\right| \\
\text { subject to: } & a_{k}=0 \quad \text { for } k \in \mathcal{I}^{*} \\
& H(z) \text { is stable }
\end{aligned}
$$

By substituting (9b) into $H\left(e^{j \omega}\right)$ in $(9 \mathrm{a}),(9 \mathrm{~b})$ is eliminated and the numerator of $H\left(e^{j \omega}\right)$ becomes

$$
a\left(e^{j \omega}\right)=\sum_{\substack{k=0 \\ k \notin \mathcal{I}^{*}}}^{n} a_{k} e^{-j k \omega}=\hat{\boldsymbol{a}}^{T}(\hat{\boldsymbol{c}}(\omega)-j \hat{\boldsymbol{s}}(\omega))
$$

where vector $\hat{\boldsymbol{a}}$ collects the nonzero coefficients of $\boldsymbol{a}$, and $\hat{\boldsymbol{c}}(\omega)$ and $\hat{\boldsymbol{s}}(\omega)$ are vectors respectively with cosine and sine components associated with the nonzero $a_{k}$ 's. Note that the coefficients of denominator $b(z)$ are now treated as a part of design variables. With the notation in (3e), we can write $b\left(e^{j \omega}\right)=b_{r}(\omega)-j b_{i}(\omega)$, and the problem at hand becomes

$$
\begin{aligned}
& \underset{\hat{\boldsymbol{a}}, \boldsymbol{b}}{\operatorname{minimize}} \underset{\omega \in \Omega}{\operatorname{maximize}}\left|\frac{\hat{\boldsymbol{a}}^{T}(\hat{\boldsymbol{c}}(\omega)-j \hat{\boldsymbol{s}}(\omega))}{b_{r}(\omega)-j b_{i}(\omega)}-H_{d}(\omega)\right| \text { (10a) } \\
& \text { Subject to: } b(z) \text { is stable }
\end{aligned}
$$

Note that vector $\hat{\boldsymbol{a}}$ is essentially the same as vector $\boldsymbol{a}$, but with sparsity removed hence its dimension is reduced at least 
by $K$. As such, (10) is a standard formulation for nonsparse minimax design of stable IIR filters. There are many methods in the literature that deal with the problem in (10), see Ch. 16 of [17] and the references cited there for details. Once the solution vectors $\hat{\boldsymbol{a}}$ and $\boldsymbol{b}$ of problem (10) are obtained, we construct a sparse vector $\boldsymbol{a}$ whose components with indices not in $\mathcal{I}^{*}$ are filled in with those of $\hat{\boldsymbol{a}}$ and zeros for $k \in \mathcal{I}^{*}$.

\section{Phase 3-Performance enhacement}

The design may be considered complete as long as both phases 1 and 2 are done. But is there a simple "follow-up" step that for instance generates a filter having more sparse coefficients without considerably degrading approximation accuracy? To address the question, let us take a look at the linear representation of $H\left(e^{j \omega}\right)$ given by (2) in connection with the design steps in phases 1 and 2: Phase 1 essentially identifies a low-dimensional subspace spanned by basis functions $\left\{w_{k}(\omega), k \in \mathcal{I}^{*}\right\}$ and claims that the design of a stable IIR filter with satisfactory performance can be carried out in that low-dimensional subspace; and Phase 2 actually carries out the design that yields a sparse IIR filter. From this perspective, the question arised above becomes one of identifying a subspace of even lower dimension in which the final design shall be performed. A natural way to further reduce the dimension of the working subspace is to examine the design result from phase 2 , namely the optimized coefficient vector $\hat{\boldsymbol{a}}$, and approximate it with a sparse vector $\hat{\boldsymbol{a}}^{*}$ so as to increase the sparsity of the IIR filter. Evidently, additional steps need to be done to ensure the filter's optimality within the reduced subspace. Specifically, design phase 3 consists of two steps as follows.

1) Apply hard-thresholding with an appropriate threshold to vector $\hat{\boldsymbol{a}}$ obtained from phase 2 to generate more zero coefficients. Bear in mind there is always a tradeoff between the degree of sparsity and performance of the filter in terms of approximation accuracy in both magnitude and phase responses. The critical result of this step is an augmented index set $\mathcal{I}^{* *}$ that contains set $\mathcal{I}^{*}$ (obtained from phase 1) as a subset.

2) Redo optimization (9) with $\mathcal{I}^{*}$ in (9b) replaced by $\mathcal{I}^{* *}$.

\section{A Design EXAMPle}

We illustrate the algorithm described in Sec. III with an example of designing a lowpass IIR filter of order $(n, r)=$ $(26,2)$ with a sparsity lower bound $K=6$ for coefficients $\left\{a_{k}\right\}$. The desired frequency response is given in terms of normalized passband edge $\omega_{p}=0.4 \pi$, stopband edge $\omega_{a}=$ $0.45 \pi$ and passband group delay 16 . The largest magnitude of poles is required to be less than 0.95 .

To prepare basis functions $\left\{w_{k}(\omega)\right\}$, a nonsparse IIR filter of order $(26,2)$ with the same design specifications was designed using the method in Ch. 16 of [17]. The denominator obtained is given by

$$
b(z)=1-0.44115170 z^{-1}+0.9 z^{-2}
$$

which was used to construct $\left\{w_{k}(\omega)\right\}$ using (3a)-(3e). A total of $L=200$ grid points evenly placed over $\left[0, \omega_{p}\right] \bigcup\left[\omega_{a}, \pi\right]$ were used to form set $\Omega$. With $\delta=0.05$, SeDuMi was used to solve problem (8). The solution vector $\boldsymbol{a}$ contains six coefficients whose magnitudes are less than $10^{-8}$. The indices of these practically zero coefficients are identified to form the index set $\mathcal{I}^{*}=\{2,4,9,11,23,25\}$. We stress that in case the number of (practically) zero coefficients does not meet the required sparsity bound $K$, constant $\delta$ in (8c) may be adjusted to expand the feasible region so as to include more sparse solutions. With $\mathcal{I}^{*}$ identified, phase 2 was performed to design a sparse IIR filter with $K=6$. The coefficients of numerator $a(z)$ are shown in the left column in Table 1, the denominator was found to be

$$
\hat{b}(z)=1-0.43970895 z^{-1}+0.9 z^{-2}
$$

To carry out phase 3, a hard-thresholding with threshold 0.0035 was applied to the coefficients $\left\{a_{k}\right\}$ obtained from phase 2 that yielded two more zeros as seen in the augmented index set $\mathcal{I}^{* *}=\{2,4,6,7,9,11,23,25\}$. This index set was used in the second step in phase 3 to solve problem (9) where $\mathcal{I}^{*}$ was replaced by $\mathcal{I}^{* *}$. The optimized $a^{*}(z)$ with 8 zero coefficients is shown in the right column in Table 1 , and the optimized denominator is given by

$$
b^{*}(z)=1-0.43800965 z^{-1}+0.902 z^{-2}
$$

The magnitude of the two poles of the optimized $H^{*}(z)=$ $a^{*}(z) / b^{*}(z)$ was found to be 0.9497 , hence the filter is stable. The maximum passband ripple, minimum stopband attenuation, and relative maximum ripple in passband group delay were found to be $0.0255,31.7625 \mathrm{~dB}$, and 0.1416 , respectively. The performance of the IIR filter obtained from phase 2 was quite comparable with that of $H^{*}(z)$, hence $H^{*}(z)$ is considered a favorable design as it is more sparse. The amplitude response of sparse IIR filter $H^{*}(z)$ (solid line) is depicted in Fig. 1. For comparison, an equivalent nonspase lowpass IIR filter of order $(n=18, r=2)$ with the same design specifications (except the passband group delay which was set to 13 for best performance) was designed using a wellestablished method described in Ch. 16 of [17]. The reason to compare $H^{*}(z)$ with a nonsparse IIR filter of order $(18,2)$ is because they have same number of nonzero coefficients. The maximum passband ripple, minimum stopband attenuation, and relative maximum ripple in passband group delay of the nonsparse IIR filter were found to be 0.0444, $27.0292 \mathrm{~dB}$, and 0.2557 . The largest magnitude of the poles was 0.9466 . The amplitude response of the equivalent nonsparse IIR filter (dashed line) is shown in Fig. 1.

It is interesting to note that the denominator $b(z)$ in (11) produced by a nonsparse design for constructing basis functions is practically the same as the denominators obtained in phases 2 and 3 as seen in (12) and (13). As a matter of fact, the relative difference in poles between $b(z)$ and $\hat{b}(z)$ and between $b(z)$ and $b^{*}(z)$ was only $7.8 \times 10^{-4}$ and $2.2 \times 10^{-3}$, respectively. This justifies the linear representation of $H\left(e^{j \omega}\right)$ with a fixed $b(z)$ as the foundation of the new design algorithm. 


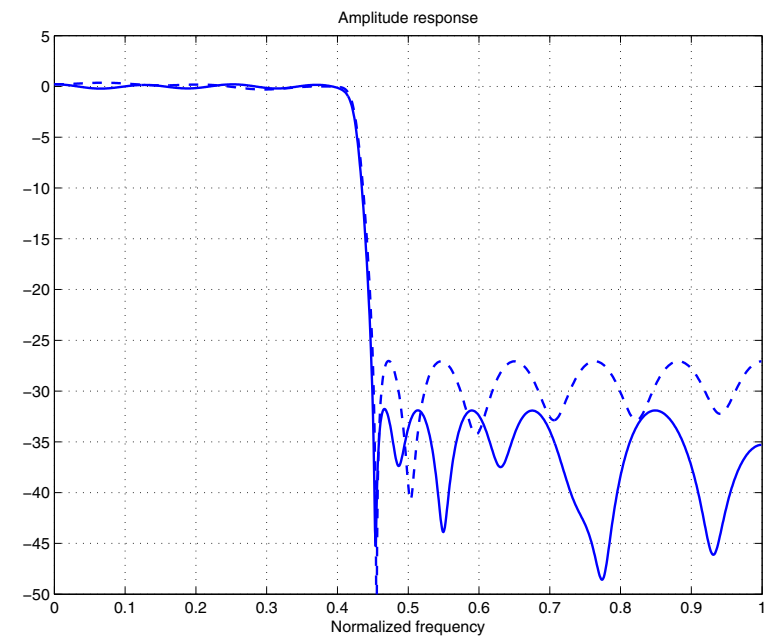

Fig. 1. Amplitude responses of the sparse IIR filter (solid line) and an equivalent nonsparse IIR filter (dashed line).

Comparison of the sparse IIR filter with a minimax linearphase FIR was also made. With $\omega_{p}=0.4 \pi$ and $\omega_{a}=0.45 \pi$, a 59-tap equiripple FIR filter was designed using the ParksMcClellan algorithm to achieve an amplitude response comparable to that of $H^{*}(z)$ designed above. The phase response of the FIR filter is perfectly linear, but its group delay is 29 compared with 16 offered by the sparse IIR filter. Moreover, with 59 taps the FIR filter requires more multiplications and additions per output relative to the sparse IIR filter which has only 18 nonzero coefficients in $a^{*}(z)$ and 2 non-unity coefficients in $b^{*}(z)$.

Based on the numerical evidence given above, we see that sparse IIR filters have the potential to offer good filtering performance as well as implementation efficiency.

\section{CONCLUSIONS}

We have presented a linear representation for stable IIR filters and, based on this, developed a new algorithm for the design of sparse IIR filters with guaranteed stability. An example is presented to illustrate the design steps of the proposed algorithm and demonstrate its good performance relative to its nonsparse IIR and FIR counterparts.

\section{REFERENCES}

[1] J. T. Kim, W. J. Oh, and Y. H. Lee, "Design of nonuniformly spaced linear-phase FIR filters using mixed integer programming," IEEE Trans. Signal Processing, vol. 44, no. 1, pp. 123-126, Jan. 1996.

[2] J. L. H. Webb and D. C. Munson, Jr., "Chebyshev optimization of sparse FIR filters using linear programming with an application to beam forming," IEEE Trans. Signal Processing, vol. 44, no. 8, pp. 1912-1922, Aug. 1996.

[3] D. Mattera, F. Palmieri, and S. Haykin, "Efficient sparse FIR filter design," Proc. ICASSP, vol. II, pp. 1537-1540, Orlando, FL, May 2002.

[4] O. Gustafsson, L. S. De Brunner, V. De Brunner, and H. Johansson, "On the design of sparse half-band like FIR filters," Proc. 41st Asilomar Conf., pp. 1098-1102, Nov. 2007.

[5] D. Wei, "Non-convex optimization for the design of sparse FIR filters," Proc. 15th IEEE Workshop on Statistical Signal Processing, pp. 117120, Cardiff, UK, Sept. 2009.
TABLE I

COEFFICIENTS OF $a(z)$ FROM PHASE 2 (LEFT COLUMN) AND FROM PHASE 3 (RIGHT COLUMN)

\begin{tabular}{|r|r|}
\hline 0.010392851102970 & 0.010036395187765 \\
0.017116960822615 & 0.015646826495216 \\
0 & 0 \\
0.005030800830541 & 0.003323342301283 \\
0 & 0 \\
0.007417943302547 & 0.008114566505141 \\
-0.000001867894394 & 0 \\
-0.003408484954507 & 0 \\
-0.007738790632433 & -0.006517428535895 \\
0 & 0 \\
0.020340709998230 & 0.023141230949229 \\
0 & 0 \\
-0.023613854872256 & -0.027680623773149 \\
-0.033053080229689 & -0.027024000127553 \\
0.052507098113062 & 0.042556154643593 \\
0.200704762712400 & 0.207983936192666 \\
0.357458937038486 & 0.349500928736363 \\
0.400358031290358 & 0.407323106379040 \\
0.316804799636574 & 0.315491540932951 \\
0.171495341811609 & 0.171347025547514 \\
0.027590628329020 & 0.032645254558257 \\
-0.012835200844937 & -0.016448691169804 \\
-0.017963977929487 & -0.014784668904078 \\
0 & 0 \\
0.010935099950532 & 0.010963543735549 \\
0 & 0 \\
-0.004853370866795 & -0.005343831411609 \\
\hline
\end{tabular}

[6] T. Baran, D. Wei, and A. V. Oppenheim, "Linear programming algorithms for sparse filters design," IEEE Trans. Signal Processing, vol. 58, no. 3, pp. 1605-1617, March 2010.

[7] W.-S. Lu and T. Hinamoto, "Digital filters with sparse coefficients," Proc. IEEE ISCAS, pp. 169-172, Paris, May 2010.

[8] W.-S. Lu and T. Hinamoto, "Two-dimensional digital filters with sparse coefficients," Multidimensional Systems and Signal Processing, vol. 22, no. 1-3, pp. 173-189, 2011.

[9] W.-S. Lu and T. Hinamoto, "Minimax design of stable IIR filters with sparse coefficients," Proc. ISCAS, pp. 398-401, Rio de Janeiro, May 2011.

[10] C.-C. Tseng and S.-L. Lee, "Design of sparse digital differentiator using orthogonal matching parsuit method," Proc. 54th IEEE MWSCAS, Seoul, Korea, Aug. 2011

[11] C. Rusu and B. Dumitrescu, "Iterative reweighted $l_{1}$ design of sparse FIR filters," Signal Processing, vol. 92, pp. 905-911, 2012.

[12] A. Jiang and H. K. Kwan, "Efficient design of sparse FIR filters in WLS sense," Proc. ISCAS, pp. 41-44, Seoul, Korea, May 2012.

[13] W.-S. Lu and T. Hinamoto, "Variable fractional delay FIR filters with sparse coefficients," Proc. ISCAS, pp. 782-785, Seoul, Korea, May 2012.

[14] E. J. Candés and M. B. Wakin, "An introduction to compressive sampling," IEEE Signal Processing Magazine, pp. 21-30, March 2008.

[15] S. Mallat, A Wavelet Tour of Signal Processing - The Sparse Way, 3rd ed., Academic Press, 2008.

[16] R. Niemistö and B. Dumitrescu, "Simplified procedures for quasiequirripple IIR filter design," IEEE Signal Processing Letters, vol. 11, no. 3, pp. 308-311, Mar. 2004.

[17] A. Antoniou and W.-S. Lu, Practical Optimixation: Algorithms and Engineering Applications, Springer 2007. 